\title{
Experiencia multicultural y su relación con la convivencia escolar
}

\section{Multicultural experience and its relationship with the coexistence in the school}

DOI: http://dx.doi.org/10.17981/cultedusoc.12.1.2021.18

Recibido: 18 de abril de 2020. Aceptado: 08 de septiembre de 2020. Publicado: 10 de febrero de 2021

\author{
Jhorman Jesid Coronado-Peña (1) \\ Universidad del Quindío. Armenia (Colombia) \\ coronadojhorman1995@gmail.com
Ángela Alexandra Estrada-Mosquera (1)
Universidad de Pamplona. Pamplona (Colombia) \\ aaexandraestrada@outlook.com \\ Leidy Nohemí Torres-Mosquera \\ Universidad de Pamplona. Pamplona (Colombia) \\ leidytorres694@hotmail.com
}

Para citar este artículo:

Coronado-Peña, J., Estrada-Mosquera, A. y Torres-Mosquera, L. (2021). Experiencia multicultural y su relación con la convivencia escolar. Cultura, Educación y Sociedad, 12(1), 341-364. DOI: http://dx.doi.org/10.17981/cultedusoc.12.1.2021.22

\section{Resumen}

El presente artículo tiene como propósito analizar la experiencia multicultural y su relación con la convivencia en el aula de estudiantes en grado quinto. Esta investigación cualitativa se desarrolló mediante un estudio de caso, utilizando la observación directa y participante y la entrevista como técnicas de recolección de información soportado en el diario de campo y guión de entrevista. Los datos obtenidos fueron interpretados mediante un análisis de contenido. Se encontró que la diversidad cultural influye en los procesos de interacción e integración en la escuela al ser esto un factor de rechazo para aquellos estudiantes culturalmente distintos; incluso se presenta exclusión entre ellos mismos. No obstante, algunos educandos poseen la capacidad adaptativa de crear lazos de amistad para disipar diferencias y, existe un reconocimiento de que el compartir con otras culturas posibilita aprender de manera íntegra. Así mismo, la institución educativa se preocupa por la educación intercultural, pero es necesaria la creación de estrategias para garantizar la inmersión de todos sus estudiantes en los eventos escolares. Se concluye que, por la influencia de la multiculturalidad en la convivencia escolar, se hace necesario cambios pedagógicos y didácticos en la enseñanza escolar como fundamento de la adaptación a la realidad escolar y al intercambio de experiencias valoracionales para la comprensión de las singularidades del otro.

Palabras clave: Multiculturalidad; convivencia escolar; diversidad; interculturalidad

\section{Abstract}

The purpose of this article is to analyze the multicultural experience and its relationship with coexistence in the classroom of fifth grade students. This qualitative research was developed through a case study, using direct and participant observation and the interview as information gathering techniques supported by the field diary and interview script. The data obtained were interpreted through content analysis. It was found that cultural diversity influences the processes of interaction and integration in the school as this is a rejection factor for those culturally different students; there is even exclusion among themselves. However, some students have the adaptive capacity to create bonds of friendship to dissipate differences, and there is a recognition that sharing with other cultures makes it possible to learn with integrity. Likewise, the educational institution cares about intercultural education, but it is necessary to create strategies to guarantee the immersion of all its students in school events. It is concluded that, due to the influence of multiculturalism in school coexistence, pedagogical and didactic changes in school teaching are necessary as a basis for adaptation to the school reality and the exchange of evaluative experiences for the understanding of the singularities of the other.

Keywords: Multiculturalism; school coexistence; diversity; interculturality 


\section{INTRODUCCIÓN}

En Colombia distintas culturas coexisten en una misma zona geográfica y social debido a la globalización y otras variantes como la crisis migratoria que cada día se contempla con más frecuencia (Vélez, 2013; VanAlstine, Cox y Roden, 2015; Rosenthal, Ramírez \& Bernardo, 2019). Sobre estas características se puede entender el concepto de multiculturalidad que no solo afronta los estereotipos asociados a cada cultura, sino que promueve la convivencia armoniosa entre las mismas. De acuerdo con Imbernón (2000) y Zubieta, Sosa y Beramendi (2011) la multiculturalidad no se puede comprender como una simple actuación para facilitar la inserción social de personas con otra procedencia cultural, sino como la aceptación de realidades plurales que enriquezcan la convivencia.

Desde esta óptica, el hablar de una educación de calidad implica dimensionar aspectos tan importantes como es la diversidad cultural presente en las escuelas, su articulación con la convivencia escolar y sus componentes relacionados. De acuerdo con Rodríguez y Fernández (2018), "la diversidad cultural en los centros educativos es una realidad incuestionable" (p. 2); por lo cual, es común que se presenten distintos estilos de vida en las aulas de clases; es decir que, se pueden encontrar diversidad en creencias, lazos culturales, hábitos, entre otros; generando en algunos momentos, ambientes conflictivos por falta de tolerancia y aceptación entre las diferencias del grupo. Estos conflictos dados por las diferencias culturales, según Palomino y Dagua (2009) y Howarth y Andreouli (2014) conllevan en ocasiones a generar baja autoestima, aislamiento, bajas calificaciones, enfrentamientos entre pares, entre otros. Así mismo, Arslam (2016) y Banerjee, Byrd y Rowley (2018) sostienen que aquellos estudiantes víctimas de una u otra forma de rechazo, por lo general presentan un rendimiento escolar más bajo al promedio estándar.

Tal situación, ha llevado a que investigaciones como las de Soraire (2012), VísquezMoren, Valenzuela-González y Compeán-Aguilar (2015), Rodríguez y Fernández (2018) y Beniscelli, Riedemann y Stang (2019), en países como Argentina, México, Chile, España, entre otros, indaguen por saber cómo los centros educativos abordan la multiculturalidad; cuáles son sus alcances y sus limitaciones. Para Colombia se registran estudios orientados a comprender los sentidos y significados de los ambientes escolares culturalmente diversos existentes en algunas regiones de país (Olivar, León y Román, 2017; Izquierdo, 2018).

Sin embargo, los hallazgos no son alentadores pues se observa la prevalecía de prácticas homogéneas en las escuelas; poco interés por la inclusión y creación de estrategias multiculturales, lo que constituye una barrera en el progreso de una educación donde se aborde la pluriculturalidad latente en las aulas (Beniscelli et al., 2019). Así mismo se hace valioso seguir analizado la configuración de la subjetividad de cada sujeto, por cuanto cada individuo se construye a partir de la relación que pueda establecer con sus compañeros en espacios de retroalimentación de experiencias (Olivar et al., 2017). En consecuencia es importante la exploración de propuestas que permitan adentrase en investigaciones cualitativas organizadas bajo un sistema de vivencias situacionales, contextualizadas y territoriales (Izquierdo, 2018), para develar lo fundamental de deconstruir aquellos procesos mentales de diferenciación 
que intervienen en los educandos, representando vivencias de violencia y dolor en el trayecto personal y familiar, consecuencias de la desigualdad de distribución (Soraire, 2012).

Es así como se hace necesario que la mirada de la educación cambie, por tanto, es importante ir en búsqueda de modelos adecuados a la heterogeneidad (agentes de todas las razas, culturas y etnias) vigentes en las escuelas (Alves, 2005; Özturgut, 2011; Syahbudin \& Hanafi; 2017; García-González, 2021); como por ejemplo adecuar más espacios para contribuir ofertas sociales, a fin de reconocer y dar importancia a las culturas ajenas y a las propias de su territorio. Resulta beneficioso proyectar la educación de las próximas generaciones alrededor de una coexistencia armoniosa en entornos multiculturales y plurales donde se eliminen ciertos tabúes, la violencia y la exclusión; consecuencias de formas de pensamiento intolerante y excluyente relacionadas con lo que cada individuo desarrolla en su vida (Cid, Díaz, Pérez, Torruella y Milady, 2008; Rodríguez, 2010; Muhammad, 2016), según haya sido las identificaciones fundantes del ser y su propio sistema de valores e ideales; los mismos pueden ser afianzados en la educación inicial (Cáceres, Granada y Pomés, 2018).

Por lo anterior, es pertinente buscar formas de analizar las variantes que se consideren puedan afectar la convivencia escolar y, observar la relación de los conflictos vivenciados con las diferencias socioculturales tales como la etnia, el género, las costumbres, las culturas, la religión, la forma de pensar o actuar, situación económica e incluso vestimenta, pues esto permitirá la creación de estrategias para promover en los niños la adaptación a un medio cultural que genere un desarrollo integral, en la cual se destaque la interacción e integración de la diferencia cultural; pero con los mismos fines y derechos educativos (Jiménez y Fardella, 2015; Alismail, 2016). Por ello, mediante el presente artículo se busca analizar la experiencia multicultural y su relación con la convivencia en el aula de los estudiantes de grado quinto.

\section{Metodología}

El artículo se fundamenta en un enfoque cualitativo de acuerdo con Guerrero (2016), el cual sostiene que la investigación cualitativa busca entender "la vida social por medio de significados, desde una visión holística, es decir que trata de comprender el conjunto de cualidades que al relacionarse producen un fenómeno determinado" (p. 2). Así mismo, se emplea la metodología de estudio de caso ya que esta "es el estudio de la particularidad y de la amplitud de un caso singular, para llegar a comprender su actividad en circunstancias importantes" (Stake, 1999, p. 11). La investigación de la cual deriva el artículo permitió identificar las siguientes etapas:

En primer, se realizó una aproximación al contexto escolar en una Institución Educativa Oficial de básica primaria en Cúcuta (Norte de Santander, Colombia). Se hicieron observaciones directas (Rojas, 2011) en las aulas del grado 5 "A", 5 "B" y 5 "C"; con estudiantes con edades comprendidas entre 10 y 11 años, los cuales representan un grado avanzado de conciencia en las actitudes y conocimiento del entorno, que les hace ser más independientes y sentirse plenamente capaces de hacer las cosas por sí mismos (Gay, Díaz, Frid, González y Olivieri, 2009). 
De esta manera, se observó el ambiente escolar en las actividades propuestas por los maestros titulares del grado durante ese periodo. Las actividades comprendieron exposiciones, evaluaciones en pareja y talleres que requerían la integración e interacción de todos sin excepción. La finalidad de estas consistió en lograr la participación en conjunto de cada uno de los estudiantes sin ninguna discriminación alguna; comprendiendo la diversidad que se encuentra en un contexto social, en este caso, escolar. A partir de esto, se analizaron las conductas de los estudiantes frente a las dinámicas principalmente colectivas, los conflictos de exclusión que se generan en dicha participación y la incidencia de alguna diferencia cultural.

Como segunda etapa se realizó un proceso de observación participante (Jociles, 2016), en la cual se desarrolló una interacción con todo el salón por parte de los investigadores; realizando un conversatorio con preguntas cortas que permitió detallar cada uno de los aspectos que demarcan para ellos alguna diferencia, por ejemplo, si sabían qué era ser extranjero, qué religiones conocían, qué era un estrato socioeconómico, entre otras. Por otro lado, con el fin de saber si se conocían un poco entre pares; se realizó un taller de parejas en donde se representó la vida del otro como propia, destacando aspectos como país o región de procedencia, creencias y gustos particulares.

Finalmente, se efectuó la selección de informantes clave. Fueron seleccionados estratégicamente seis estudiantes, según los objetivos de estudio y sus características multiculturales observadas a lo largo de los momentos de la investigación —nacionalidad, religión, género, estrato socioeconómico, color de piel, etnia- (Mendieta, 2015). A partir de ello, se llevó a cabo una entrevista de tipo personal, empleando para el logro de la información un guion de entrevista (Díaz-Bravo, Torruco-García, Martínez-Hernández y Varela-Ruiz, 2013) avalado por dos expertos en el tema; los cuales determinaron que el instrumento reunía las condiciones de validación para su aplicación, al presentar concordancia y pertinencia con los objetivos propuestos en la investigación. Dentro de la entrevista se contemplaron once interrogantes con relación al tema de estudio. Es importante aclarar que, la participación se registró mediante el consentimiento informado de cada estudiante; donde se aclaró la confidencialidad de la información suministrada.

Respecto al registro de información y su análisis; los datos observados tanto en la primera y segunda etapa fueron registrados en un diario de campo siguiendo un orden de forma completa, precisa y detallada (Monistrol, 2007; Hernández, 2017) y, esta información junto con lo descrito por los informantes clave en la entrevista, fue tratada mediante un análisis de contenido (Arandes, 2013), que conlleva a la agrupación de información según los términos relacionados, siguiendo una idea central; permitiendo la codificación, categorización y ordenamiento de información; ayudando a descifrar datos importantes para un análisis crítico y reflexivo de acuerdo a cada etapa vivenciada (San Martín, 2014).

\section{Resultados y Discusión}

Durante el acercamiento al ambiente escolar, las observaciones directas develaron la influencia que tiene la diversidad cultural con la interacción misma del aula, al hallarse rechazo a estudiantes de acuerdo a sus características socioeconómicas, religión, género, 
nacionalidad, color de piel y etnia, cuando se invitaba a actividades colectivas en la cual se requería la integración e interacción entre los estudiantes para las distintas dinámicas pedagógicas y didácticas propuestas por el maestro titular. Aquel alumno presente con los rasgos descritos era susceptible a no formar grupos de trabajo o no ser seleccionados como primera opción para los equipos. Es importante precisar que el número de estudiantes por aula dificultaba la colaboración en el orden para cualquier actividad.

A lo anterior Hurtado (2015) señala que, este tipo actividades grupales promueven la disminución de "índices de violencia dentro de la comunidad escolar y posibilita herramientas para la convivencia" (p. 34). De este modo, el trabajo grupal suscita la integración de la diversidad de personalidades. Sin embargo, Cordoba (2020) recomienda que esta clase de estrategias sean abordadas desde una mirada holística; buscando la equidad, justicia y mitigación de conflicto mediante la inquietud socio-cultural; dato importante ya que en el grupo de estudiantes analizado se detalló actitudes individualistas bastante notables, lo que hace que la educación sea fragmentada y la integración y sana convivencia para el respeto de las diferencias sea difícil de construir.

En el caso de la observación participante, se pudo apreciar que los estudiantes reconocieron algunas características de un extranjero ("personas de otra nacionalidad"), las religiones ("individuos con ideologías de un ser todo poderoso creador") y los estratos socioeconómicos ("condición que da a un sujeto cierto nivel riqueza en la sociedad”). Además, los estudiantes poseían algunos conocimientos sobre las características culturales de sus pares. Sin embargo, este reconocimiento del uno al otro no necesariamente era en pro de una sana convivencia para la comprensión de la vida de sus compañeros y el dimensionamiento de cuerpos sintientes, sino que por el contrario, era usado para menoscabar la dignidad del estudiante. Estos datos podrían explicar el comportamiento de los discentes en la primera etapa observacional; en donde sus acciones son apáticas frente a la interacción con estudiantes culturalmente distintos.

A este respecto la Comisión Nacional de Derechos Humanos (2012) y Saleem et al. (2016) plantean que la discriminación es dibujada por patrones socioculturales tradicionalmente aprendidos y estos rasgos muchas veces obedecen a la incomprensión y temor frente a las diferencias; por lo que es necesario educar en la igualdad y paz sociocultural, puesto que ello construye una convivencia armónica de toda la diversidad.

En la última etapa se encontró lo siguiente frente a los once interrogantes dispuestos en el guión de la entrevista:

A la primera pregunta: ¿Con qué género te identificas y por qué? Todos los entrevistados demostraron seguridad a la hora de contestar, manifestando el género con el cual se identifican. No se observó alguna "desorientación sexual" ni duda acerca de los géneros existentes. Los entrevistados narraron que hay diferencias notorias entre el comportamiento de los niños y las niñas e incluso, insisten que nacieron con el género asignado según designio de Dios, es decir que Dios los hizo hombres y mujeres (Tabla 1). Lo anterior demuestra la claridad que poseen los estudiantes con respecto a su orientación sexual definida. 
TABLA 1.

Fragmentos y clasificación de las respuestas brindadas por los estudiantes frente a la pregunta 1: ¿Con qué género te identificas?

\begin{tabular}{|c|c|c|c|}
\hline Sujeto & Unidad de análisis & Categoría & Subcategoría \\
\hline 1 & $\begin{array}{l}\text { "Soy del género femenino. Porque soy } \\
\text { delicada". }\end{array}$ & Diversidad & Género \\
\hline 2 & $\begin{array}{l}\text { "Soy del género masculino. Porque los } \\
\text { hombres se diferencian por tener una } \\
\text { capacidad más dura y fuerte. Las mujeres } \\
\text { son delicadas". }\end{array}$ & Diversidad & Género \\
\hline 3 & $\begin{array}{l}\text { "Me siento y me identifico con el género } \\
\text { masculino porque soy hombre y así me hizo } \\
\text { Dios". }\end{array}$ & Diversidad & Género \\
\hline 4 & $\begin{array}{l}\text { "Me identifico con el género masculino } \\
\text { porque soy hombre y así nací". }\end{array}$ & Diversidad & Género \\
\hline 5 & “Soy hombre porque así nací”. & Diversidad & Género \\
\hline 6 & $\begin{array}{l}\text { "Me identifico con el género masculino } \\
\text { porque soy hombre y así me creó Dios". }\end{array}$ & Diversidad & Género \\
\hline
\end{tabular}

Fuente: Elaboración propia.

Hernández y Jaramillo (2003) y García-Piña (2016) afirman que, en esta cultura, generalmente un niño comienza a adquirir sus primeros conocimientos con relación a la sexuación humana mediante otros medios distintos a los de su propio cuerpo; aprenden a distinguir uno de otros (niños y niñas) a partir del aspecto físico y comportamental que representen dentro de su entorno, pero en sí, aun no conciben lo que es el sexo. Es con el paso del tiempo donde empiezan a descubrir que una u otra persona tienen cuerpos distintos de acuerdo con su sexo. El anterior planteamiento permite deducir que los estudiantes entrevistados en el rango de edad en el que están poseen la capacidad de determinar su género con claridad y madurez y tienen conocimiento de la identidad sexual, lo que les permite tener libertad de decisión.

Para la pregunta dos: ¿Por qué existen diferentes orientaciones sexuales? Los entrevistados plantearon que esta situación se presenta porque la mayoría siente inconformidad con el sexo biológicamente asignado (Tabla 2). Estas percepciones son un poco inquietantes pues aún los estudiantes no conciben de forma natural la diversidad existente alrededor; incluso cuando actualmente, de acuerdo con Larios y Cuevas (2016) la cotidianidad está tejida de posturas socioculturales diversas, lo que debería crear un ambiente que reconozca una sociedad con una amplia gama de comportamientos sexuales no propiamente heterosexuales. Es allí, donde parte la multiculturalidad desde de sus inicios como la agrupación de una serie de movimientos sociales; grupos racialmente oprimidos, feministas, LGTB, revolucionarios o reformistas, en su proceso de institucionalización a fin de dar un giro a la diversidad no como un problema sino como un conjunto de recursos para estructurar las relaciones interculturales (Dietz, 2007; León, 2011); pues aún existen ciertas conductas e ideas latentes en los imaginarios sociales en la cual la diversidad sexual es tomada como algo indeseable o que debe ser reprimido y castigado (Chávez, Zapata, Petrzelová y Villanueva, 2018). 
TABLA 2.

Fragmentos y clasificación de las respuestas ofrecidas por los estudiantes frente a la pregunta 2: ¿Por qué existen diferentes orientaciones sexuales?

\begin{tabular}{|c|c|c|c|}
\hline Sujeto & Unidad de análisis & Categoría & Subcategoría \\
\hline 1 & $\begin{array}{l}\text { "Porque no están de acuerdo con su cuerpo. Hay niñas que les } \\
\text { gusta pelear y hay niños que no". }\end{array}$ & Inconformidad & Género \\
\hline 2 & $\begin{array}{l}\text { "Hay personas que no les gusta el sexo que tienen y se identifican con } \\
\text { el sexo opuesto. Hay otras personas que les gusta el mismo sexo". }\end{array}$ & Inconformidad & Género \\
\hline 3 & $\begin{array}{l}\text { "Hay hombres que no les gustan las mujeres. Creen que son gay. } \\
\text { Les agrada sentirse así y que las personas les digan así". }\end{array}$ & Desorientación & Género \\
\hline 4 & $\begin{array}{l}\text { "Hay hombres que se creen y se sienten mujeres. Porque hay } \\
\text { personas que les gusta eso". }\end{array}$ & Desorientación & Género \\
\hline 5 & "No lo sé por qué son así". & Desinformación & Género \\
\hline 6 & $\begin{array}{l}\text { "Porque así se acostumbraron. Están equivocadas, Dios no los creó } \\
\text { así. Las personas son las que empiezan hacer cosas malas". }\end{array}$ & Rechazo & Género \\
\hline
\end{tabular}

Fuente: Elaboración propia.

Por otro lado, se presentó un entrevistado con desinformación de la temática; lo que generó desinterés e incluso apatía al tema. Esta situación tiene relación con el planteamiento de la Comisión Nacional de Derechos Humanos (2012) y Cornejo (2018) al destacarse cierta indiferencia hacia estos temas tras la poca información o formación que poseen los estudiantes sobre una cuestión en particular; lo que genera incomprensión y rechazo frente a lo no conocido o a las diferencias.

Frente al tercer interrogante: ¿Te sientes cómodo al tratar con alguien que tenga orientación sexual diferente? Se pudo apreciar que los entrevistados en su totalidad se sienten bien al tratar con personas con orientación sexual diferente, dándoles un trato digno de amabilidad e incluso de cariño y amor. No se observaron conductas de rechazo ni discriminación aun cuando no están de acuerdo con ciertas cosas; se puede apreciar entonces, que los niños respetan las diferencias y asumen con madurez el valor ofrecido a una persona independientemente de su orientación sexual (Tabla 3).

TABLA 3.

Fragmentos y clasificación de las respuestas brindadas por los estudiantes frente a la pregunta 3: ¿Te sientes cómodo al tratar con alguien que tenga orientación sexual diferente?

\begin{tabular}{llll}
\hline Sujeto & \multicolumn{1}{c}{ Unidad de análisis } & Categoría & Subcategoría \\
\hline 1 & "Me siento normal y los acepto". & Aceptación & Género \\
2 & "Sí, los acepto. Me siento cómodo". & Aceptación & Género \\
3 & "Me siento bien y normal. Los trato bien”. & Aceptación & Género \\
4 & $\begin{array}{l}\text { "Tengo un amigo que se siente mujer. Trato a todos por } \\
\text { igual. Lo trato bien porque es una persona igual a todos". } \\
\text { "Me siento cómodo al tratar con personas homosexuales. }\end{array}$ & Agualdad & Género \\
5 & $\begin{array}{l}\text { Los trato normal". } \\
\text { "Me siento bien. Las trataría con amor y cariño". }\end{array}$ & Aceptación & Género \\
\hline
\end{tabular}

Fuente: Elaboración propia. 
Esto es acorde a una de las misiones principales de la educación multicultural: "la reivindicación del valor de la diferencia etnia o cultura, así como en la lucha por la pluralización de las comunidades socialmente marginadas o rechazadas" (Dietz, 2005, p. 28). Aguado (2011) afirma:

La escuela sigue siendo una vía insustituible para lograr objetivos valiosos en sociedades que defienden principios de participación y justicia social. El enfoque intercultural se propone como una mirada hacia la diversidad de los estudiantes, familias y comunidades que reconoce dicha diversidad y asume un compromiso por la igualdad de oportunidades y una escuela buena para todos (p. 23).

A la pregunta: ¿Qué opinas de que haya estudiantes venezolanos en el salón? Se pretendía abordar la información de los estudiantes acerca de la crisis migratoria en Colombia. Pareciera a simple vista no ser un dato relevante para explicárselo a un niño, sin embargo, a través de lo observado y lo analizado en las respuestas, se encontró que la desinformación de la situación actual del vecino país fomenta ente ellos tomar conceptos que extraen desde el contexto, es decir lo escuchado a su alrededor, adaptando nombres despectivos para referirse a ellos o asegurando que vinieron a hacer daño (Tabla 4).

TABLA 4.

Fragmentos y clasificación de las respuestas brindadas por los estudiantes frente a la pregunta 4: ¿Qué opinas de que haya estudiantes venezolanos en el salón?

\begin{tabular}{|c|c|c|c|}
\hline Sujeto & Unidad de análisis & Categoría & Subcategoría \\
\hline 1 & $\begin{array}{l}\text { "Me siento normal al momento de convivir } \\
\text { con estudiantes venezolanos porque todos } \\
\text { tienen derecho a la educación". }\end{array}$ & $\begin{array}{l}\text { Convivencia en } \\
\text { el aula }\end{array}$ & Nacionalidad \\
\hline 2 & $\begin{array}{l}\text { "Es mejor convivir con otros compañeros de } \\
\text { diferente cultura porque así se obtiene más } \\
\text { conocimiento de otros lugares". }\end{array}$ & $\begin{array}{l}\text { Convivencia en } \\
\text { el aula }\end{array}$ & Interculturalidad \\
\hline 3 & $\begin{array}{l}\text { "Todos somos iguales y la diferencia es que } \\
\text { son de otra cultura, por la situación del } \\
\text { vecino país es entendible que otros niños } \\
\text { lleguen a buscar oportunidades y a recibir } \\
\text { educación". }\end{array}$ & Multiculturalidad & Igualdad \\
\hline 4 & $\begin{array}{l}\text { "Es normal y bueno que otros niños } \\
\text { venezolanos se incluyan a la escuela porque } \\
\text { tienen las mismas oportunidades y derecho a } \\
\text { la educación". }\end{array}$ & $\begin{array}{l}\text { Convivencia en } \\
\text { el aula }\end{array}$ & Igualdad \\
\hline 5 & $\begin{array}{l}\text { "Les dicen groserías; veneco" (y otras } \\
\text { palabras despectivas de lenguaje vulgar que } \\
\text { por respeto al lector no se transcribieron). }\end{array}$ & $\begin{array}{l}\text { Convivencia en } \\
\text { el aula }\end{array}$ & Igualdad \\
\hline 6 & $\begin{array}{l}\text { "A los niños venezolanos los tratan mal; } \\
\text { por la situación del país vecino muchos han } \\
\text { tenido que salir para otros lugares a buscar } \\
\text { mejores oportunidades y algunas familias no } \\
\text { tienen dinero suficiente para la educación". }\end{array}$ & Multiculturalidad & Vulnerabilidad \\
\hline
\end{tabular}

Fuente: Elaboración propia. 
En tal sentido, es fundamental invitar a la tolerancia y el respetar unos con otros a pesar de las diferencias, con el fin de dejar a un lado prejuicios hacia grupos culturales en particular y, que así emerja y se teja una sana convivencia. Esto se podría lograr creando espacios para hablar o explicarles a los estudiantes el porqué de estas situaciones que se están viviendo y así promover la igualdad.

No obstante autores como Cigarroa, Rojas, Evangelista y Saldívar (2016) y Robles y Hernández (2019) aluden que esta problemática no es fácil de afrontar pues no se proporcionan los recursos necesarios para abordarlo en las escuelas y hay falta de interés por parte de los docentes, directivos, padres de familia e incluso de los mismos estudiantes; además que cada centro educativo según la Comisión Española de Ayuda al Refugiado (2016) y Marchesi y Hernández (2019) varía en mayor y menor media de acuerdo a sus particularidades; por ejemplo la cantidad de estudiantes presentes en las aulas, la diversidad de los mismos según la ubicación del centro, entre otros; lo que dificulta educar en una convivencia intercultural y el aprendizaje mutuo. Sin embargo, el panorama devela un desafío en las escuelas que valdrá la pena porque posibilitará una sociedad democrática, equitativa y mayor desarrollo del país (Duran y Parra, 2014; Castillo-Briseño, 2015).

Cada informante expresó que independientemente que sus compañeros tuvieran otra nacionalidad, eso no les impide a ellos estudiar; para ellos todos tienen derecho a la educación. Una de las respuestas indicó que es bueno compartir con otras culturas y aprender de ellas, no obstante, esto tiende a convertirse en contradictorio tras las dificultades analizadas en la convivencia escolar en el aula. Desde la perspectiva de Mena y Hunneuss (2017), el buen trato entre los actores escolares es la base de una sana convivencia. La especificación dada desde la multiculturalidad existente en un aula de clase tiene gran incidencia en la convivencia escolar, por cuanto al tomar como ejemplo la crisis migratoria actual y la inserción de niños venezolanos al sistema educativo colombiano; y en este caso en el colegio seleccionado para la investigación, se observó discriminación y rechazo que en grandes rasgos podría convertirse en un problema social si no se educa para vivir y convivir en espacios interculturales.

Así mismo, los entrevistados expresaron en la pregunta cinco: ¿Has observado que alguna vez se ha discriminado a alguien por ser de diferente nacionalidad? ¿qué opinas sobre eso?, en la institución se presentan casos de discriminación y aislamiento por ser de diferente nacionalidad (confirmando así la discriminación observada por los investigadores). Los estudiantes manifiestan, además, que esas actitudes no son adecuadas y que no han participado de ellas (Tabla 5). Sin embargo, se vivencian esas situaciones en donde es necesaria la intervención del docente quien cumple un papel fundamental en el marco de la sana convivencia educativa. Por lo anterior, la Organización de las Naciones Unidas para la Educación, la Ciencia y la Cultura (UNESCO, 2005) con el fin de formar al maestro para el trabajo en el aula multicultural, propone una cartilla: "Diversidad Cultural", como guía para:

[...] proveer a los docentes de una fuente de formación sobre el amplio tema de la diversidad cultural y potenciar una mirada educativa desde y hacia la diversidad; contribuyendo a la difusión del conocimiento sobre el tema, al presentar casos de estudio con ejemplos concretos para que los docentes los adecuen a su trabajo pedagógico (p. 8). 
TABLA 5.

Fragmentos y clasificación de las respuestas brindadas por los estudiantes frente a la pregunta 5: ¿Has observado que alguna vez se ha discriminado a alguien por ser de diferente nacionalidad? ¿Qué opinas sobre eso?

\begin{tabular}{llll}
\hline Sujeto & \multicolumn{1}{c}{ Unidad de análisis } & Categoría & Subcategoría \\
\hline & $\begin{array}{l}\text { "Lo único que les dicen a los niños de otra nacionalidad es } \\
\text { veneco y no he visto un mal trato hacia ellos. No se les debería } \\
\text { decir así solo porque vienen de otro país y utilizan otras } \\
\text { expresiones". }\end{array}$ & $\begin{array}{l}\text { Diversidad } \\
\text { cultural }\end{array}$ & Discriminación \\
& $\begin{array}{l}\text { "Está mal discriminar a alguien, porque todos somos personas } \\
\text { y todas tienen los mismos derechos y oportunidades". }\end{array}$ & $\begin{array}{l}\text { Convivencia } \\
\text { en el aula }\end{array}$ & Igualdad \\
3 & $\begin{array}{l}\text { "Creo que es bueno tratar bien a los compañeros de otras } \\
\text { nacionalidades". }\end{array}$ & Multiculturalidad & Integración \\
4 & $\begin{array}{l}\text { "Está mal discriminar a alguien; todos somos iguales". } \\
\text { "He observado discriminación a los niños venezolanos, con } \\
\text { comentarios feos como veneco y no se relacionan casi con } \\
\text { ellos". }\end{array}$ & Multiculturalidad & Igualdad \\
"Hacemos juegos para conocer otras culturas, pintamos, & aula & Discriminación \\
hacemos dibujos, lenguas y música". & Interculturalidad & Integración \\
\hline
\end{tabular}

Fuente: Elaboración propia.

TABLA 6.

Fragmentos y clasificación de las respuestas brindadas por los estudiantes frente a la pregunta 6: ¿Qué actividades se han realizado en clase para compartir y dar a conocer diferentes culturas a nivel nacional o local?

\begin{tabular}{|c|c|c|c|}
\hline Sujeto & Unidad de análisis & Categoría & Subcategoría \\
\hline 1 & $\begin{array}{l}\text { "Las actividades que han realizado en el aula son para } \\
\text { conocer otras culturas como la región Insular, Orinoquía, } \\
\text { las costumbres. Hicimos una cartelera con la cual } \\
\text { teníamos que exponer todo lo de las culturas de las } \\
\text { regiones". }\end{array}$ & $\begin{array}{l}\text { Convivencia en el } \\
\text { aula }\end{array}$ & $\begin{array}{l}\text { Integración } \\
\text { cultural }\end{array}$ \\
\hline 2 & $\begin{array}{l}\text { "Las actividades que se hacen en la institución son } \\
\text { la semana cultural, donde se observa carteles que } \\
\text { representan las diferentes culturas, con los platos típicos y } \\
\text { fotos del vestuario según la región". }\end{array}$ & Multiculturalidad & $\begin{array}{l}\text { Integración } \\
\text { cultural }\end{array}$ \\
\hline 3 & $\begin{array}{l}\text { "En música nos enseñan el folklor para conocer y } \\
\text { aprender de otras culturas y nacionalidades. En el aula } \\
\text { nos han enseñado sobre las razas andinas, mestizas, } \\
\text { afrodescendientes". }\end{array}$ & Interculturalidad & $\begin{array}{l}\text { Interacción e } \\
\text { integración } \\
\text { cultural }\end{array}$ \\
\hline 4 & $\begin{array}{l}\text { "En el área de sociales nos enseñan los tipos de piel, } \\
\text { culturas, lengua y acento para tener conocimiento de la } \\
\text { diversidad cultural". }\end{array}$ & Multiculturalidad & $\begin{array}{l}\text { Interacción e } \\
\text { integración } \\
\text { cultural }\end{array}$ \\
\hline 5 & $\begin{array}{l}\text { "Hacemos juegos para conocer otras culturas: pintamos, } \\
\text { hacen dibujos". }\end{array}$ & $\begin{array}{l}\text { Convivencia en el } \\
\text { aula }\end{array}$ & Integración \\
\hline 6 & "Nos enseñan sobre los diferentes idiomas". & Multiculturalidad & $\begin{array}{l}\text { Interacción } \\
\text { cultural }\end{array}$ \\
\hline
\end{tabular}

Fuente: Elaboración propia. 
Según las respuestas dadas al interrogante seis: ¿Qué actividades se han realizado en clase para compartir y dar a conocer diferentes culturas a nivel nacional o local?, la institución se preocupa por la integración y educación de la cultura, por cuanto se realizan actividades donde los niños no solo conocen la diversidad del país sino que se involucran en programaciones de interacción cultural e incluso el aprendizaje de otros idiomas, con el fin de internacionalizar la educación abordando distintas variantes como la música, arte y culinaria (Tabla 6).

Este tipo de estrategias metodológicas planeadas en la institución analizada, permiten la equidad y aceptación entre compañeros (Rodríguez y Tejada, 2018; Lotero-Franco, Restrepo-Segura y Arroyave-Taborda, 2021) por cuanto se promueven procesos reflexivos alrededor de las diferencias, y se contribuye al fortalecimiento de una escuela incluyente (Figueroa, Gutiérrez y Velázquez, 2017; Rangel-Ledezma, Arzola-Franco, González-Ortiz, y Conchas-Ramírez, 2021); tras el disfrute de experiencias de aprendizaje de calidad, donde se establecen relaciones centradas en el respeto y la justicia social (López-Vélez, 2018; Guzmán y Álvarez, 2020). Es de esta manera como es preciso la creación de escenarios que respondan a las necesidades de educativas.

Es importante señalar también que el centro educativo examinado, no solo realiza actividades para proyectos en el aula sino también institucionales para involucrar la comunidad y dar participación a los padres de familia; incorporando una pedagogía inter y multicultural. Según el Fondo de las Naciones Unidas para la Infancia (UNICEF, 2008):

El aprendizaje es un proceso que dura toda la vida. Un enfoque de la educación basado en los derechos humanos busca crear las oportunidades para que todos los niños alcancen sus capacidades óptimas a lo largo de la niñez y, después de estas (p. 29).

En este sentido, se desarraiga la posibilidad de conductas discriminatorias en el futuro; de aquí radica la importancia de culturizar desde temprano una futura sociedad de igualdad. Por su parte, entendemos por pluralismo, como lo expresa Taylor citado por Pinedo (2013): El pluralismo trasciende del hecho de solo la coexistencia de diversas culturas en un sitio determinado, es también la acción de que estas se comprendan y construyan una dinámica enriquecedora complementaria orientada al mejoramiento del entorno. Tanto los niños como la institución reconocen el valor significativo de enriquecerse de cultura general como un paso importante para crear vías de diálogo y mediación de conflictos asociados a la diferencia, mejorando la integración escolar, la capacidad de tolerancia y la sana convivencia. De esta forma, la escuela representa más que un contexto donde se obtienen conocimientos meramente académicos alrededor de unas áreas básicas, un espacio con experiencias valoracionales tanto éticas y morales, que nutren, construyen y complementan dichos conocimientos y fortalece la percepción del alumnado en aras de una convivencia determinada.

Por otro lado, los informantes expresaron a la pregunta: ¿Qué trato se les da a compañeros que pertenecen a otras culturas?, que el trato no es el más adecuado en personas con culturas diversas pues hay acciones de discriminación por parte de compañeros. Se indicó en sí, que todo ser humano es igual, por lo cual están en desacuerdo frente a estas situaciones (Tabla 7). También, en algunas ocasiones se pudo observar buena convivencia 
en el aula evidenciada en la capacidad adaptativa de muchos niños para crear lazos de amistad orientadas a disipar la diferencias. De acuerdo con Almaguer y García (2014):

En las relaciones interculturales se establece una relación basada en el respeto a la diversidad y el enriquecimiento mutuo; sin embargo, no es un proceso exento de conflictos, estos se resuelven mediante el respeto, el diálogo, la escucha mutua, la concertación y la sinergia (p. 17).

TABLA 7.

Fragmentos y clasificación de las respuestas brindadas por los estudiantes frente a la pregunta 7: ¿Qué trato se les da a compañeros que pertenecen a otras culturas?

\begin{tabular}{|c|c|c|c|}
\hline Sujeto & Unidad de análisis & Categoría & Subcategoría \\
\hline 1 & $\begin{array}{l}\text { "A veces se tratan bien y a veces no, } \\
\text { porque los tratan como niños normales y } \\
\text { a veces los discriminan". }\end{array}$ & $\begin{array}{l}\text { Convivencia } \\
\text { en el aula }\end{array}$ & Discriminación \\
\hline 2 & $\begin{array}{l}\text { "El trato que se le da a los compañeros de } \\
\text { otra cultura es un trato de desprecio como } \\
\text { si fueran otra cosa, un amigo dice que } \\
\text { lo tratan y se dirigen a él como veneco o } \\
\text { mocho". }\end{array}$ & $\begin{array}{l}\text { Convivencia } \\
\text { en el aula }\end{array}$ & Bullying \\
\hline 3 & $\begin{array}{l}\text { "A los niños pertenecientes a otras } \\
\text { culturas les dicen cosas feas". }\end{array}$ & $\begin{array}{l}\text { Convivencia } \\
\text { en el aula }\end{array}$ & Bullying \\
\hline 4 & $\begin{array}{l}\text { "Todos somos diferentes y es válido que } \\
\text { tengamos gustos distintos. No me gusta } \\
\text { que traten a las personas con desigualdad } \\
\text { cultural mal". }\end{array}$ & Diversidad cultural & Interacción cultural \\
\hline 5 & $\begin{array}{l}\text { "No nos juntamos con ellos porque a veces } \\
\text { no tienen plata para ir a los paseos". }\end{array}$ & Interculturalidad & Estrato socioeconómico \\
\hline 6 & $\begin{array}{l}\text { "A veces los tratan mal por ser de otra } \\
\text { religión". }\end{array}$ & $\begin{array}{l}\text { Convivencia en el } \\
\text { aula }\end{array}$ & Igualdad \\
\hline
\end{tabular}

Fuente: Elaboración propia.

Es así como las observaciones en el aula detallaron discriminación a estudiantes con características particulares, pero a su vez - aunque en una medida baja - una actitud de interacción e incluso integración entre la diversidad presente en la escuela.

Respecto a la pregunta: ¿Te han excluido de actividades escolares por no dar aporte económico? Los estudiantes cuentan desde su experiencia que en ocasiones no han podido participar de actividades escolares por no dar el aporte económico requerido. Para algunos de sus compañeros esta situación es frecuente al no disponer de recursos suficientes, pues son de estrato socioeconómico bajo; de hecho, la ubicación de la institución se encuentra en un contexto donde coexisten estratos bajos y asisten estudiantes en situación de vulnerabilidad (Tabla 8).

Frente a lo anterior, parece entonces que el factor económico tiene gran incidencia en el proceso de formación aun cuando este sea gratuito; incluso, Pérez (2000) menciona que "la escuela juzga a todos y otorga a cada uno un lugar dentro de las jerarquías escolares" (p. 1). Los padres hacen esfuerzos para que los niños asistan a las instituciones educativas, no obstante, hay actividades que requieren suministrar aportes de tipo monetario que en muchas ocasiones no está al alcance de los estudiantes. 
TABLA 8.

Fragmentos y clasificación de las respuestas brindadas por los estudiantes frente a la pregunta 8: ¿Te han excluido de actividades escolares por no dar aporte económico?

\begin{tabular}{|c|c|c|c|}
\hline Sujeto & Unidad de análisis & Categoría & Subcategoría \\
\hline 1 & $\begin{array}{l}\text { "No me he sentido excluida, siempre participo. No sé de } \\
\text { mis compañeros". }\end{array}$ & Interculturalidad & Integración \\
\hline 2 & $\begin{array}{l}\text { "Una vez no pude ir a una excursión porque no pagué } \\
\text { a tiempo. El día de la excursión yo llegué preparado y } \\
\text { fui a pagar y no me dejaron, porque no pude pagar a } \\
\text { tiempo. Entonces me dejaron ahí, sentí un dolor en el } \\
\text { pecho casi con ganas de llorar". }\end{array}$ & Integración & Desmotivación \\
\hline 3 & $\begin{array}{l}\text { "Yo siempre participo, mis papás me dan para pagar. } \\
\text { Algunos de mis compañeros no pagan porque los } \\
\text { padres no tienen plata para pagar actividades como las } \\
\text { interclase y el uniforme deportivo". }\end{array}$ & Integración & Interacción \\
\hline 4 & $\begin{array}{l}\text { "No me he sentido excluido, pero hay compañeros que } \\
\text { van a la práctica del campeonato, pero no participan } \\
\text { porque no pagan la boleta". }\end{array}$ & Integración & Desigualdad \\
\hline 5 & "Yo siempre pago. Hay compañeros que no pagan". & Integración & Desigualdad \\
\hline 6 & $\begin{array}{l}\text { "Yo pago, pero algunos compañeros no pueden pagar } \\
\text { porque trabajan para ayudarle a los padres". }\end{array}$ & Integración & Desigualdad \\
\hline
\end{tabular}

Fuente: Elaboración propia.

Por su parte, uno de los entrevistados compartió la experiencia de haber participado en una recolección de dinero para ayudar a un compañero que no tenía los recursos económicos para adquirir el uniforme de su interclase. Este tipo de acciones son parte de pedagogía intercultural, la cual tiene en su naturaleza el promover acciones que vayan en pro de la comprensión de las diferencias culturales (González y Machado, 2011; Ghazarian \& Youhne, 2015; Lee, Poch, Smith, Kelly \& Leopold, 2018); busca "Adecuar las condiciones y los procesos de aprendizaje, en sus más diversos aspectos y sin excluir ninguno de ellos, a las características culturales específicas de los niños" (Lennon, 2016, p. 351). Por lo cual, se hace crucial que de manera amónica y sinérgica se trabaje desde los directivos, los maestros y los padres de familias por garantizar la participación de los estudiantes en cada uno de los eventos y actividades pertinentes a la educación necesaria que organiza el plantel educativo.

En la pregunta nueve: ¿Hay niños que no se integran en las actividades de la institución?, se evidenció que existen distintos factores por el cual existen niños que no se integran en eventos escolares. Como se mencionó en la pregunta ocho, el aspecto económico tiene gran incidencia, pero también a ello se suma el hecho de algunos estudiantes tímidos para relacionarse; un ejemplo de ello es una niña que no realiza actividades deportivas pues no le gusta ser observada por sus compañeros debido a su contextura. Así mismo también las diferencias culturales cohíben al niño para adaptarse a actividades grupales, debido a la exclusión de sus compañeros (Tabla 9). A este respecto, Sánchez (2015) manifiesta que: 
La inteligencia interpersonal es la capacidad de entender a los demás e interactuar eficazmente con ellos. Incluye la sensibilidad a expresiones faciales, la voz, los gestos y posturas y la habilidad para responder. Se encuentra presente en actores, políticos, buenos vendedores y docentes exitosos, entre otros. La tienen los niños que disfrutan trabajar en grupo, que son convincentes en sus negociaciones con pares, mayores y que entienden al compañero (p. 8).

TABLA 9.

Fragmentos y clasificación de las respuestas brindadas por los estudiantes frente a la pregunta 9: ¿Hay niños que no se integran en las actividades de la institución?

\begin{tabular}{lllc}
\hline \multicolumn{1}{c}{ Sujeto } & \multicolumn{1}{c}{ Unidad de análisis } & Categoría & Subcategoría \\
\hline 1 & $\begin{array}{l}\text { "Son muy penosos y no les gusta convivir con los demás". } \\
\text { "Porque hay otros compañeros que forman grupos para }\end{array}$ & Inclusión & Desmotivación \\
2 & $\begin{array}{l}\text { molestar. Las actividades son para enseñar y aprender y no } \\
\text { para dar malos ejemplos". }\end{array}$ & Inclusión & Bullying \\
& $\begin{array}{l}\text { "Hay una compañera que es muy creída y no le gusta } \\
\text { ajuntarse con nadie, le gusta hacer las cosas sola y no }\end{array}$ & Integración & Apatía \\
& $\begin{array}{l}\text { socializa con los compañeros". } \\
\text { "No tienen actitud y porque no tienen dinero". }\end{array}$ & Convivencia & Desmotivación \\
5 & "Porque no tienen dinero". & Interacción & Desmotivación \\
6 & "Porque no traen los materiales". & Integración & Desmotivación \\
\hline
\end{tabular}

Fuente: Elaboración propia.

Es importante favorecer por parte de la escuela la inteligencia interpersonal de su alumnado, a través de metodologías pedagógicas y didácticas que faciliten la relación y comunicación de sus educandos; por cuanto la exclusión a veces puede ser dada desde el mismo estudiante y no por otros, tal como se observó con la niña que no realizó actividades deportivas.

Para la pregunta: ¿Te has sentido de alguna forma discriminado? ¿por qué?, es importante tener presente que al momento de realizar la selección de los informantes se tomó en cuenta que por lo menos un estudiante de cada salón presentara alguna diferencia cultural, sea de nacionalidad, etnia, estrato socioeconómico e incluso religión, con el fin de que relatara a través de su experiencia cómo era el trato recibido, cuáles situaciones había vivenciado desde la experiencia cultural, cómo se sentían y su opinión al respecto; a lo cual respondieron que efectivamente se presentaban casos de discriminación y aislamiento en los grupos de trabajo; los llaman con nombres peyorativos (Tabla 10).

Aun cuando los entrevistados tenían una posición de negación frente a estos actos, también asumieron una postura de tolerancia y aceptaron que en ocasiones han participado de actos de discriminación hacia otros compañeros. Sin embargo, quienes han realizado estas acciones han reflexionado al observar el daño causado. En tal sentido, aprender a convivir debe convertirse en un pilar fundamental de la educación, para solidificar la interculturalidad, contribuyendo en la mejora de la convivencia escolar, el respeto, la toleración y el diálogo. Es importante entonces tratar de mitigar las acciones de discriminación y aún más cuando esta torna a ser prolongada ya que, conforme a Pinheiro (2005), Krieger (2014) y Williams, Lawrence, Davis y Cecilia (2019), esto afecta el desarrollo físico, cognitivo y social al modificar o perturbar el sistema nervioso e inmune. 
TABLA 10.

Fragmentos y clasificación de las respuestas brindadas por los estudiantes frente a la pregunta 10: ¿Te has sentido de alguna forma discriminado? ¿por qué?

\begin{tabular}{|c|c|c|c|}
\hline Sujeto & Unidad de análisis & Categoría & Subcategoría \\
\hline 1 & $\begin{array}{l}\text { "No me he sentido discriminada, pero algunos compañeros sí } \\
\text { porque tiene un color de piel diferente y porque vienen de otro } \\
\text { lugar". }\end{array}$ & Inclusión & Bullying \\
\hline 2 & $\begin{array}{l}\text { "Sí me han discriminado, porque al principio me decían veneco y } \\
\text { mocho". }\end{array}$ & Integración & Discriminación \\
\hline 3 & $\begin{array}{l}\text { "Sí me han discriminado por mi color de piel. Al principio me } \\
\text { colocaba a pelear porque me decían negro caliche, y ya me } \\
\text { acostumbré y me siento normal". }\end{array}$ & Discriminación & Bullying \\
\hline 4 & $\begin{array}{l}\text { "No me he sentido discriminado, al principio solo me decían } \\
\text { venezolano y ya no me dicen así". }\end{array}$ & Interculturalidad & Integración \\
\hline 5 & $\begin{array}{l}\text { "No me he sentido discriminado. Solo me dicen que el color de mi } \\
\text { cabello es bonito y ya". }\end{array}$ & Inclusión & Igualdad \\
\hline 6 & $\begin{array}{l}\text { "No me han discriminado, solo hay una compañera que me dice } \\
\text { malas palabras y me trata mal, diciéndome cállese la geta". }\end{array}$ & Convivencia & Discriminación \\
\hline
\end{tabular}

Fuente: Elaboración propia.

Finalmente, el interrogante once señalaba: ¿Cómo actúan tus compañeros cuando se generan actividades grupales?, a ello, los estudiantes indicaron que normalmente no logran obtener un equipo de trabajo; por lo cual trabajan solos. Estas narraciones ratifican lo observado en las etapa uno y dos de la investigación, en la cual, se pudo contemplar que en el aula cuando se generan trabajos grupales, los equipos se conforman de estudiantes que tienen mejores relaciones entre sí, quedando excluidos los que con frecuencia no se relacionan por no tener la habilidad o por ser para los niños alguien diferente (Tabla 11).

TABLA 11.

Fragmentos y clasificación de las respuestas brindadas por los estudiantes frente a la pregunta 11: ¿Cómo actúan tus compañeros cuando se generan actividades grupales?

\begin{tabular}{|c|c|c|c|}
\hline Sujeto & Unidad de análisis & Categoría & Subcategoría \\
\hline 1 & $\begin{array}{l}\text { "Hacen mucho ruido y siempre se hacen los mismos amigos, y otros } \\
\text { compañeros quedan solos y así quedan trabajando". }\end{array}$ & $\begin{array}{l}\text { Convivencia } \\
\text { en el aula }\end{array}$ & Desmotivación \\
\hline 2 & $\begin{array}{l}\text { "Empiezan a gritar y a decir unos a otros: yo me hago con usted; } \\
\text { hagamos nosotros no más. Yo me quedo solo, y otros compañeros se } \\
\text { quedan solos también porque no les gusta hacerse en grupo porque } \\
\text { molestan mucho". }\end{array}$ & $\begin{array}{l}\text { Convivencia } \\
\text { en el aula }\end{array}$ & Desigualdad \\
\hline 3 & $\begin{array}{l}\text { "La profesora nos baja la nota por hacer desorden, nos dicen que nos } \\
\text { hagamos con el que queramos y a veces la profesora nos organiza los } \\
\text { grupos y no nos gusta así porque nos toca a veces con los más cansones". }\end{array}$ & $\begin{array}{l}\text { Convivencia } \\
\text { en el aula }\end{array}$ & Desmotivación \\
\hline 4 & $\begin{array}{l}\text { "Hacen desorden porque les da pereza levantar la silla y la arrastran } \\
\text { por todo el salón y por eso hacen ruido. Algunos compañeros y yo nos } \\
\text { quedamos sin grupo y trabajamos solos". }\end{array}$ & $\begin{array}{l}\text { Convivencia } \\
\text { en el aula }\end{array}$ & Desmotivación \\
\hline 5 & "Nos hacemos con los mismos compañeros y algunos quedan solos". & $\begin{array}{l}\text { Convivencia } \\
\text { en el aula }\end{array}$ & Desigualdad \\
\hline 6 & $\begin{array}{l}\text { "Siempre se hacen los mismos para trabajar y cuando alguno queda solo } \\
\text { la profesora lo pone a trabajar en algún grupo". }\end{array}$ & $\begin{array}{l}\text { Convivencia } \\
\text { en el aula }\end{array}$ & Integración \\
\hline
\end{tabular}

Fuente: Elaboración propia. 
Esta posición preferencial en los equipos de trabajo es común en las aulas de clase, por lo cual es necesaria la intervención del maestro para forjar mejores relaciones e implementar estrategias para disminuir los conflictos y, generar aceptación, por cuanto es esencial fomentar o promover un entorno rico en compañerismo y en sentido de pertenencia. Así el trabajo en equipo resulta ser una acción que fomenta el intercambio de experiencias y el crecimiento personal, creando una oportunidad de generar interculturalidad.

La indisciplina es otro factor mencionado por los informantes que dificulta el desarrollo de las actividades en grupo. Giner y Onieva (2009), Gotzens-Busquets, Cladellas-Pros, Clariana-Muntada y Badia-Martín (2015) y Tique-Gutiérrez et al. (2018) expresan que este tipo de actitudes comportamentales perturban la tranquilidad del acto educativo y afecta el rendimiento académico. Por ello, es importante que el docente desarrolle métodos didácticos para captar la atención de sus estudiantes de una manera rápida, práctica y diversa, dejando a un lado situaciones monótonas las cuales aburren a los estudiantes, al presentarse siempre una enseñanza rutinaria.

\section{CONCLUSIONES}

Después de analizados los resultados obtenidos se concluye que la multiculturalidad presente en la escuela y su influencia en la convivencia da cuenta de la importancia que la institución educativa posee de transformar sus procesos metodológicos de enseñanza; tanto pedagógicos como didácticos y brindar ambientes escolares conforme a las realidades escolares, donde se susciten el intercambio de experiencias valoracionales para la comprensión de las singularidades del otro; creando una oportunidad de generar interculturalismo para coexistir en espacios culturalmente diversos. Además, es importante que estas acciones sean dadas no solo desde los maestros, sino de manera integrada con los directivos y padres de familia.

La diversidad cultural no debería tomarse únicamente como un asunto étnico o migratorio, se deben especificar todas las variantes que implican la pluralidad en el aula de clase, por cuanto es importante develar las significaciones generadas a la multiculturalidad con una premisa importante que surge a través de la observación del contexto y es su relación con la convivencia del aula.

La convivencia escolar, es una construcción cotidiana de vivencias en los principales escenarios de participación como los centros educativos; por tanto, la diversidad en esta no debe constituirse como un obstáculo, sino como un conjunto de recursos para estructurar las relaciones interculturales, su integración e interacción. Es así necesaria una pedagogía intercultural que promueva respetar y tolerar diferencias, y así mismo pueda desterrar prejuicios y estereotipos asociados; generando una relación armoniosa, que no solo dé lugar a un aprendizaje significativo, sino que, a través del incremento de las aulas pluralizadas en Colombia, pueda darse un progreso en la multiculturalidad. 


\section{REFERENCIAS}

Aguado, O. (2011). El enfoque intercultural en la búsqueda de buenas prácticas escolares. Latinoamericana de Inclusión Educativa, 5(2), 23-42. Recuperado de http:// www.rinace.net/rlei/numeros/vol5-num2/art1.pdf

Alismail, H. (2016). Multicultural Education: Teachers' Perceptions and Preparation. Journal of Education and Practice, 7(11), 139-146. Recuperado de https://files.eric. ed.gov/fulltext/EJ1099450.pdf

Almaguer, G. \& García, R. (2014). Interculturalidad en salud. [Online]. Recuperado de http://www.dged.salud.gob.mx/contenidos/dged/descargas/docs/InterculturalidadSalud.pdf

Alves, F. (2005). La formación de los profesores ante los retos de la sociedad multicultural. Revista Castellano-Manchega de Ciencias Sociales, 7, 19-148. https://doi. org/10.20932/barataria.v0i7.240

Arandes, T. (2013). El análisis de contenido como herramienta de utilidad para la realización de una investigación descriptiva. Un ejemplo de aplicación práctica utilizado para conocer las investigaciones realizadas sobre la imagen de marca de España y el efecto país de origen. Provincia, 29, 135-173. Disponible en http://www.saber.ula.ve/ handle/123456789/38452

Arslam, G. (2016). Relationship between sense of rejection, academic achievement, academic efficacy, and educational purpose in high school students. Egitim ve Bilim, 41(183), 293-304. https://doi.org/10.15390/EB.2016.5562

Banerjee, M., Byrd, C. \& Rowley, S. (2018). The Relationships of School-Based Discrimination and Ethnic-Racial Socialization to African American Adolescents' Achievement Outcomes. Social sciences, 7(10), 1-19. https://doi.org/10.3390/socsci7100208

Beniscelli, L., Riedemann, A. y Stang, F. (2019). Multicultural y, sin embargo, asimilacionista. Paradojas provocadas por el currículo oculto en una escuela con alto porcentaje de alumnos migrantes. Calidad en la educación, 50, 393-423. http://dx.doi. org/10.31619/caledu.n50.522

Cáceres, Z., Granada, A. y Pomés, C. (2018). Inclusión y Juego en la Infancia Temprana. Latinoamericana de Educación Inclusiva, 12(1), 181-199. Disponible en http://www. rinace.net/rlei/numeros/vol12-num1/art10.html

Castillo-Briseño, C. (2015). Posicionando la educación inclusiva: Una forma diferente de mirar el horizonte educativo. Educación (Costa Rica), 39(2), 123-152. http://dx.doi. org/10.15517/revedu.v39i2.19902

Chávez, M., Zapata, J., Petrzelová, J. \& Villanueva, G. (2018). La diversidad sexual y sus representaciones en la juventud. Psicogente, 21(39), 62-74. http://doi.org/10.17081/ psico.21.39.2822 
Cid, H., Díaz, M., Pérez, M., Torruella, P. \& Milady, A. (2008). Agresión y violencia en la escuela como factor de riesgo del aprendizaje escolar. Ciencia y Enfermería, 14(2), 21-30. http://dx.doi.org/10.4067/S0717-95532008000200004

Cigarroa, R., Rojas, W., Evangelista, G. y Saldívar, M. (2016). Educación inclusiva y niñez migrante: barreras a la educación de niñez de origen centroamericano en escuelas de Tapachula, Chiapas (México). Investigación en Educación, 14(1), 85-104. Recuperado de http://reined.webs.uvigo.es/index.php/reined/article/view/226/249

Comisión Española de Ayuda al Refugiado. (2016). Informe sobre discriminación de personas migrantes y refugiadas en España. [Online]. Recuperado de https://www.cear. es/wp-content/uploads/2017/02/Informe-discriminaci\%C3\%B3n.pdf

Comisión Nacional de Derechos Humanos. (2012). La discriminación y el derecho a la no discriminación. [Online]. Recuperado de http://familiasysexualidades.inmujeres.gob. $\mathrm{mx} / \mathrm{pdf} / 2$ _Cartilla_Discriminacion.pdf

Cordoba, S. (2020). Posconflicto e interculturalidad como modelo para mejorar la calidad educativa. Cultura, Educación y Sociedad, 11(1), 241-256. http://dx.doi.org/10.17981/ cultedusoc.11.1.2020.18

Cornejo, E. J. (2018). Discriminación y violencia homofóbica en el sistema escolar: estrategias de prevención, manejo y combate. Brasileira de Educação, 23, 1-24. Disponible en https://anped.org.br/site/rbe/rbe

Díaz-Bravo, L., Torruco-García, U., Martínez-Hernández, M. y Varela-Ruiz, M. (2013). La entrevista, recurso flexible y dinámico. RRIEM Revista Investigación en educación médica, 2(7), 162-167. Disponible en http:/www.riem.facmed.unam.mx:90/ojs/ index.php/riem/article/view/430

Dietz, G. (2007). Multiculturalismo: un breviario para el debate. Palabra y el hombre, 2, 38-42. Recuperado de https://cdigital.uv.mx/bitstream/handle/123456789/28714/20 0702P38.pdf?sequence=1\&isAllowed $=y$

Dietz, G. (2005). Del multiculturalismo a la interculturalidad: evolución y perspectivas. En, G. Carrera, y G. Dietz, Patrimonio inmaterial y gestión de la diversidad (Colección Cuadernos IAPH) (pp. 30-51). Sevilla: Consejería de Cultura/Junta de Andalucía.

Duran, S. \& Parra, M. (2014). Diversidad Cultural para promover el desarrollo de habilidades sociales en educación superior. Cultura, Educación y Sociedad, 5(1), 55-67. Disponible en https://revistascientificas.cuc.edu.co/culturaeducacionysociedad/article/view/995

Figueroa, Á., Gutiérrez, B. y Velázquez, L. (2017). Estrategias de inclusión en contextos escolares. Diversitas - Perspectivas en Psicología, 13(1), 13-26. https://doi. org/10.15332/s1794-9998.2017.0001.01 
García-González, V. (2021). Descolonización de los saberes del docente como base para la promoción e implementación de prácticas educativas significativas. Cultura, Educación y Sociedad, 12(1), 241-254. http://dx.doi.org/10.17981/cultedusoc.12.1.2021.16

García-Piña, C. (2016). Sexualidad infantil: información para orientar la práctica clínica. Acta Pediátrica de México, 37, 47-53. http://dx.doi.org/10.18233/APM37No1pp47-53

Gay, M., Díaz, Z., Frid, N., González, M. y Olivieri, J. (2009). La preadolescencia: un nuevo desafío para la Salud Mental infantil. Revista Pediátrica del Hospital de Niños de Buenos Aires, 51(234), 197-234. Recuperado de http://revistapediatria.com. ar/wp-content/uploads/2012/03/con288-197.pdf

Ghazarian, P. \& Youhne, M. (2013). Exploring Intercultural Pedagogy: Evidence From International Faculty in South Korean Higher Education. Studies in International Education, 19(5), 476-490. Disponible en https://journals.sagepub.com/doi/ pdf/10.1177/1028315315596580

Giner, P. y Onieva, M. (2009). ¿Qué hacer ante la indisciplina en las aulas? Formació del Professorat, 4, 1-6. Recuperado de http://cefire.edu.gva.es/sfp/revistacompartim/ arts4/20_au_indisciplina_aulas.pdf

González, P. y Machado, D. (2011). Extensión universitaria en la universalización de la Educación Superior: una mirada desde la pedagogía intercultural. Humanidades Médicas, 11(2), 274-289. Disponible en http://humanidadesmedicas.sld.cu/index. $\mathrm{php} / \mathrm{hm} / \mathrm{article/view/81}$

Gotzens-Busquets, C., Cladellas-Pros, R., Clariana-Muntada, M. y Badia-Martín, M. (2015). Indisciplina instruccional y convencional: su predicción en el rendimiento académico. Colombiana de Psicología, 24(2), 317-330. http://dx.doi.org/10.15446/rcp. v24n 2.44148

Guerrero, B. (2016). Investigación cualitativa. INNOVA Research Journal, 1(2), 1-9. https://doi.org/10.33890/innova.v1.n2.2016.7

Guzmán, N. y Álvarez, M. (2020). Aproximación teórica a las estrategias de acompañamiento en el ambiente familiar y escolar. Cultura, Educación y Sociedad, 11(1), 151-166. http://dx.doi.org/10.17981/cultedusoc.11.1.2020.11

Hernández, M. y Jaramillo, G. (2003). La educación sexual en la primera infancia. Madrid: Ministerio de Educación, Cultura y Deporte. Recuperado de http://www.codajic.org/sites/www.codajic.org/files/Guia\%20Educaci\%C3\%B3n\%20Sexual\%20en\%20 la\%20Primera\%20Infancia.pdf

Hernández, R. (2017). Reflexiones metodológicas. Estudios sobre las Culturas Contemporáneas, XXIII(4), 167-197. Recuperado de https://www.culturascontemporaneas. com/culturascontemporaneas/contenidos/09Ninossoldados.pdf 
Howarth, C. \& Andreouli, E. (2015). 'Changing the context': tackling discrimination at school and in society. International Journal of Educational Development, 41, 184191. https://doi.org/10.1016/j.ijedudev.2014.06.004

Hurtado, M. (2015). El Juego herramienta metodológica para la convivencia escolar en niños de educación básica primaria. [Tesis de pregrado]. Corporación Universitaria Lasallista, Caldas, Colombia. Recuperado de http://repository.lasallista.edu.co/dspace/bitstream/10567/1721/1/Juego_herramienta_metodologica_para_la_convivencia. pdf

Imbernón, F. (2000). Escuela y multiculturalidad. Pensamiento Educativo, 26, 153171. Recuperado de https://pensamientoeducativo.uc.cl/files/journals/2/articles/170/ public/170-416-1-PB.pdf

Izquierdo, B. (2018). Educación en contextos multiculturales: experiencia etnoeducativa e intercultural con población indígena del Resguardo Embera Chamí-Mistrató, Risaralda-Colombia. Zona Próxima, 29, 1-22. Disponible en http://hdl.handle. net/11059/8727

Jiménez, F. y Fardella, C. (2015). Diversidad y rol de la escuela. Mexicana de Investigación Educativa, 20(65), 419-441. Disponible en http://www.rmie.mx/

Jociles, R. (2016). La observación participante: ¿consiste en hablar con "informantes”? QuAderns-e, 21(1), 113-124. Recuperado de https:/www.raco.cat/index.php/QuadernseICA/article/download/317138/407207

Krieger, N. (2014). Discrimination and health inequities. International Journal of Health Services, 44(4), 643-710. Disponible en https://journals.sagepub.com/doi/pdf/10.2190/ HS.44.4.b

Larios, D. y Cuevas, J. (2016). Diversidad sexual. Colima: Universidad de Colima. Recuperado de http://www.ucol.mx/content/publicacionesenlinea/adjuntos/Diversidadsexual-y-universidad_433.pdf

Lee, A., Poch, R., Smith, A., Kelly, M. D. \& Leopold, H. (2018). Intercultural Pedagogy: A Faculty Learning Cohort. Education sciences, 8(4), 1-14. https://oi.org/10.3390/ educsci8040177

León, O. (2011). Los retos de las sociedades multiculturales: interculturalismo y pluralismo. Cuadernos Intercambio sobre Centroamérica y el Caribe, 9, 207-227. Recuperado de https://revistas.ucr.ac.cr/index.php/intercambio/article/view/2222

Lennon, L. (2016). Limitaciones y posibilidades de la pedagogía intercultural para niños indígenas. Estudios Pedagógicos, XLII(1), 339-353. https://doi.org/10.4067/S071807052016000100022

López-Vélez, A. (2018). La escuela inclusiva: el derecho a la equidad y la excelencia educativa. Bilbao: Universidad del País Vasco. Recuperado de https://web-argitalpena. adm.ehu.es/pdf/USPDF188427.pdf 
Lotero-Franco, J., Restrepo-Segura, Y. y Arroyave-Taborda, L. (2021). Diseños curriculares e inteligencia social en zonas urbanas y rurales de Colombia. Cultura Educación y Sociedad, 12(1), 255-274. http://dx.doi.org/10.17981/cultedusoc.12.1.2021.17

Marchesi, A. y Hernández, L. (2019). Cinco Dimensiones Claves para Avanzar en la Inclusión Educativa en Latinoamérica. Latinoamericana de Educación Inclusiva, 13(2), 45-56. http://dx.doi.org/10.4067/S0718-73782019000200045

Mena, M. \& Huneeus, M. (2017). Convivencia Escolar para el aprendizaje y buen trato de todos: Hacia una mejor comprensión del concepto. Cultura Educación y Sociedad, 8(2), 9-20. https://doi.org/10.17981/cultedusoc.8.2.2017.01

Mendieta, I. (2015). Informantes y muestreo en investigación cualitativa. Investigaciones andinas, 17(30), 1148-1150. Disponible en https://revia.areandina.edu.co/index.php/ IA/article/view/65

Monistrol, R. (2007). El trabajo de campo en investigación cualitativa (II). Nure Investigación, (29), 1-4. Disponible en https:/www.nureinvestigacion.es/OJS/index.php/ nure/article/view/350/341

Muhammad, M. (2016). Multicultural education in the diversity of national cultures. Qudus International Journal of Islamic Studies, 4(2), 167-185. Recuperado de https:// core.ac.uk/download/pdf/295525897.pdf

Olivar, M., León, G. y Román, M. (2017). Diversidad cultural y reconocimiento: una mirada desde los espacios universitarios. [Tesis de maestría]. Universidad de Manizales, Popayán, Colombia. Disponible en http://ridum.umanizales.edu.co:8080/xmlui/bitstream/handle/6789/3083/Olivar_Cesar_Hernan. pdf? sequence=1\&isAllowed=y

Özturgut, O. (2011). Understanding multicultural education. Current Issues in Education, 14(2), 1-11. Recuperado de http://cie.asu.edu/ojs/index.php/cieatasu/article/ view/732

Palomino, L. y Dagua, P. (2009). Los problemas de convivencia escolar: percepciones, factores y abordajes en el aula. Investigaciones UNAD, 8(2), 199-221. https://doi. org/10.22490/25391887.643

Pinedo, A. (2013). Filosofía multicultural y educación para la convivencia ciudadana. Praxis \& Saber, 4(7), 179-199. https://doi.org/10.19053/22160159.2055

Pinheiro, P. (2005). Informe mundial sobre la violencia de niños y niñas. [Online]. Recuperado de https://observatoriodelainfancia.vpsocial.gob.es/productos/pdf/informeMundialSobreViolencia.pdf

Pérez, S. (2000). La escuela frente a las desigualdades Sociales. Apuntes sociológicos sobre el pensamiento docente. Iberoamericana de educación, 23, 1-21. Recuperado de https://red.pucp.edu.pe/ridei/files/2012/05/120517.pdf 
Rangel-Ledezma, Y., Arzola-Franco, D., González-Ortiz, A. y Conchas-Ramírez, M. (2021). Manejo de conflictos y atención a las necesidades de los centros educativos en educación primaria. Cultura, Educación y Sociedad, 12(1), 133-150. http://dx.doi. org/10.17981/cultedusoc.12.1.2021.09

Robles, G. y Hernández, F. (2019). Jóvenes inmigrantes latinos en la escuela. Del rechazo escolar a la acogida en las bandas. Estudios socioeducativos, (7), 71-87. Disponible en https://revistas.uca.es/index.php/ReSed/article/view/4456/5499

Rodríguez, F. y Fernández, F. (2018). Agentes educativos y multiculturalidad en el aula. Educação e Pesquisa, 44, 1-21. http://dx.doi.org/10.1590/S16784634201844188506

Rodríguez, R. (2010). Exclusión cultural en América Latina y el Caribe. Interuniversitaria de Formación del Profesorado, 69(24), 73-86. Disponible en https://redined. mecd.gob.es/xmlui/bitstream/handle/11162/37860/ExclusionCulturalEnAmericaLatinaYElCaribe-3361185.pdf;jsessionid=1FF090129419E6C5509BA91E9087D529?s equence $=1$

Rodríguez, A. y Fernández, A. D. (2018). Agentes educativos y multiculturalidad en el aula. Educação e Pesquisa, 44, 1-21. https://doi.org/10.1590/s1678-4634201844188506

Rodríguez, R. y Tejada, C. (2018). Atención a la diversidad e inclusión educativa: implicaciones didácticas. Madrid: Asociación Accesibilidad Para Todos - La Ciudad Accesible. Recuperado de https://sid.usal.es/idocs/F8/FDO27377/iAccessibility_15. pdf

Rojas, C. (2011). Elementos para el diseño de técnicas de investigación: una propuesta de definiciones y procedimientos en la investigación científica. Tiempo de Educar, 12(24), 277-297. Recuperado de http://hdl.handle.net/20.500.11799/38942

Rosenthal, L., Ramírez, L., Levy, S. \& Bernardo, A. (2019). Polyculturalism: Viewing Cultures as Dynamically Connected and its Implications for Intercultural Attitudes in Colombia. Avances en Psicología Latinoamericana, 37(1), 133-151. https://doi. org/10.12804/revistas.urosario.edu.co/apl/a.7175

Saleem, F., English, D., Busby, D., Lambert, S., Harrison, A., Stock, M. \& Gibbons, F. (2016). The Impact of African American Parents' Racial Discrimination Experiences and Perceived Neighborhood Cohesion on their Racial Socialization Practices. Youth Adolescence, 45, 1338-1349. https://doi.org/10.1007/s10964-016-0499-x

San Martín, D. (2014). Teoría fundamentada y Atlas.ti: recursos metodológicos para la investigación educativa. Electrónica de Investigación Educativa, 16(1), 104-122. Disponible en https://redie.uabc.mx/redie/article/view/727/891

Sánchez, A. (2015). La teoría de las inteligencias múltiples en la educación. México, D.F.: Universidad Mexicana. Recuperado de https://unimex.edu.mx/Investigacion/DocInvestigacion/La_teoria_de_las_inteligencias_multiples_en_la_educacion.pdf 
Soraire, E. (2012). La diversidad cultural en la universidad: algunos mecanismos que operan en la configuración de las identidades culturales. Cuadernos FHyCS-UNJu, 42, 105-116. Disponible en http://revista.fhycs.unju.edu.ar/revistacuadernos/index. php/cuadernos/article/view/92

Stake, R. (1999). Investigacion con estudio de casos. Madrid: Morata. Recuperado de https://www.uv.mx/rmipe/files/2017/02/Investigacion-con-estudios-de-caso.pdf

Syahbudin, Z. \& Hanafi, M. (2017). The model of learning design based on islamic multicultural education to prevent conflicts of behavior. Jurnal Pendidikan Islam, 3(2), 155-168. Available: https://media.neliti.com/media/publications/229207-the-modelof-learning-design-based-on-is-cb1c4404.pdf

Tique-Gutiérrez, M., Camacho-Anaya, J., Segura-Guevara, Y., Orozco-Torregrosa, R., Ortega-Moreno, L., Iriarte-Ariza, M., Martínez-Rudas, J., Gutiérrez-Pérez, J. y Cáceres-Fernández, N. (2018). Contexto socioeconómico sobre el rendimiento académico del estudiante mediante la investigación como estrategia pedagógica. Cultura, Educación y Sociedad, 9(1), 171-180. http://dx.doi.org/10.17981/cultedusoc.9.1.2018.13

UNESCO. (2005). Diversidad Cultural: materiales para la formación docente y el trabajo de aula. [Online]. Recuperado de https://unesdoc.unesco.org/ark:/48223/pf0000151226

UNICEF. (2008). Un Enfoque de la Educación para Todos basado en los derechos humanos: marco para hacer realidad el derecho de los niños a la educación y los derechos en la educación. [Online]. Disponible en https:/unesdoc.unesco.org/ark:/48223/ pf0000158893

VanAlstine, J., Cox, S. \& Roden, D. (2015). Cultural Diversity in the United States and Its Impact on Human Development. Journal of the Indiana Academy of the Social Sciences, 18, 125-143. Disponible en https:/digitalcommons.butler.edu/jiass/vol18/ iss $1 / 10$

Vélez, T. (2013). Desplazamiento y etnicidad: fracasos del multiculturalismo en Colombia. Desacatos, 41, 155-173. Recuperado de https://desacatos.ciesas.edu.mx/index. php/Desacatos/article/download/93/27/115

Víquez-Moren, D., Valenzuela-González, J. \& Compeán-Aguilar, M. (2015). Identificación de competencias transversales para reformas curriculares: El caso de la multiculturalidad. Electrónica Educare, 19(2), 333-358. https://doi.org/10.15359/ree.19-2.19

Williams, D. R., Lawrence, J. A., Davis, B. A. \& Cecilia, V. (2019). Understanding how discrimination can affect health. Health Services Research, 54, 1374-1388. https:// doi.org/10.1111/1475-6773.13222

Zubieta, E., Sosa, F. y Beramendi, M. (2011). Adaptación Cultural, actitudes hacia el multiculturalismo y ansiedad intergrupal en población militar. Boletín de Psicología, 102, 55-69. Recuperado de https://www.uv.es/seoane/boletin/previos/N102-4. pdf 
Jhorman Jesid Coronado Peña es Licenciado en Biología y Educación Ambiental. Universidad del Quindío (Armenia, Colombia). https://orcid.org/0000-0002-8554-8088

Ángela Alexandra Estrada Mosquera es Licenciada en pedagogía infantil de la Universidad de Pamplona (Pamplona, Colombia). https://orcid.org/0000-0003-0652-7164

Leidy Nohemí Torres Mosquera es Licenciada en pedagogía infantil de la Universidad de Pamplona (Pamplona, Colombia). https://orcid.org/0000-0002-6866-0182 